\title{
Autonomic dysfunction in multiple sclerosis and other updates on recent autonomic research
}

\author{
Mitchell G. Miglis ${ }^{1} \cdot$ Srikanth Muppidi ${ }^{1}$
}

Received: 6 July 2018 / Accepted: 7 July 2018 / Published online: 16 July 2018

c) Springer-Verlag GmbH Germany, part of Springer Nature 2018

Keywords Orthostatic hypotension $\cdot$ Dysautonomia $\cdot$ Heart rate variability $\cdot$ Multiple sclerosis $\cdot$ Syncope

\section{Autonomic dysfunction in multiple sclerosis}

Multiple sclerosis (MS) is a demyelinating disorder of the central nervous system (CNS) that can result in varied neurological deficits, including motor dysfunction, sensory loss, and bladder abnormalities. Additionally, patients report significant fatigue. Although reports on the symptoms of autonomic dysfunction in patients with MS are increasing, the nature of the dysautonomia in MS remains unclear. Two recent publications have attempted to address this important topic. In an article published in Multiple Sclerosis \& Related Disorders, Damla et al. [1] measured heart rate variability (HRV) in 51 patients recently diagnosed with relapsing-remitting multiple sclerosis (RRMS) and 44 matched healthy controls. All patients were evaluated before any immunomodulatory therapy was started. Participants had echocardiograms as well as 24-h HRV analysis. Those with an abnormal echocardiogram were excluded from the study. Additionally, all patients had brain and cervical MR imaging. The authors found that patients with RRMS had lower HRV values than controls, but there was no relationship between the location of the demyelinating lesions and the severity of the HRV abnormalities. There was also no relationship between HRV and MS-specific outcome measures, such as the Expanded Disability Status Scale (EDSS) or MS Functional Composite scores. Thus, patients with recently diagnosed MS appear to have a reduction in HRV without any clear relationship to the overall disease burden at the time of diagnosis. Therefore, the clinical relevance of these findings remains unclear. Moreover, since this was a crosssectional study, it is unknown if these HRV abnormalities

Srikanth Muppidi

muppidis@stanford.edu

1 Department of Neurology, Stanford Medical Center, 213

Quarry Road, 2nd Floor, Palo Alto, CA 94304, USA worsen as patients develop further demyelinating plaque burden or as they develop a progressive form of MS.

In a different article published in Clinical Neurophysiology, Adamec et al. [2] analyzed HRV and performed standardized autonomic function testing in 40 patients with RRMS and 30 patients with progressive multiple sclerosis (PMS). Patients with PMS had worse HRV measures and a higher sudomotor index (as measured by quantitative sudomotor axon reflex testing, QSART) and Composite Autonomic Severity Score (CASS) than patients with RRMS. Additionally, the duration of disease and the EDSS positively correlated with a higher sudomotor index and total CASS. The authors concluded that patients with progressive MS have worse autonomic nervous system function, in particular sudomotor dysfunction. Given that the QSART measures post-ganglionic (i.e., peripheral) sudomotor function, an obvious question is: how is it possible that a CNS disease such as MS results in peripheral nerve dysfunction? The answer remains elusive at this time.

These two studies highlight the growing recognition of autonomic dysfunction in immune-mediated demyelinating disorders such as MS. We do not yet know if the autonomic dysfunction is a mere reflection of the underlying CNS disease affecting the central autonomic network, or if there is additional immune-mediated, perhaps peripheral, pathophysiology [3]. Also, it is not clear if autonomic dysfunction in these patients is subtle or clinically relevant. Since there are many new and relatively effective therapies for MS, it is important to know if aggressive treatment to prevent disease progression also leads to improvement in autonomic symptoms. We hope that future treatment studies of MS will include autonomic markers in order to explore this important association and to assess responsiveness to treatment. 


\section{Is all orthostatic hypotension created equal?}

The severity of orthostatic symptoms can vary substantially between patients. Some patients report few or no symptoms despite severe orthostatic hypotension $(\mathrm{OH})$, whereas other patients report significant orthostatic intolerance despite no observable changes in blood pressure (BP) or heart rate (HR) during the head-up tilt (HUT). While the definition of $\mathrm{OH}$ is well established by consensus criteria [4], various subtypes of $\mathrm{OH}$ may exist, each of which might indicate differences in pathophysiology and prognosis. In a recent article published in the Journal of Clinical Neurology, Seok et al. [5] retrospectively studied the patterns of orthostatic BP changes in a cohort of patients with $\mathrm{OH}$ who had been diagnosed with standardized autonomic testing, including a 10-min HUT test. The authors subdivided the patients with confirmed $\mathrm{OH}$ into the following categories: sustained orthostatic hypotension ( $\mathrm{sOH}$, defined as a relatively stable systolic BP without a decrease or a recovery of $\geq 10 \mathrm{mmHg}$ after decreasing $\geq 20 \mathrm{mmHg}$ within $3 \mathrm{~min}$ of HUT), progressive orthostatic hypotension ( $\mathrm{pOH}$, defined as either a continuous BP decrease of $\geq 10 \mathrm{mmHg}$ beyond $3 \mathrm{~min}$ of HUT or a stable BP without a decrease or a recovery of $\geq 10 \mathrm{mmHg}$ during the initial $5 \mathrm{~min}$, followed by a decrease of $\geq 15 \mathrm{mmHg}$ between 5 and $10 \mathrm{~min}$ of HUT), orthostatic hypotension with partial recovery $(\mathrm{OHpr}$, defined as either an incomplete recovery of $\geq 10 \mathrm{mmHg}$ in $\mathrm{BP}$-remaining $\geq 20 \mathrm{mmHg}$ below baseline-within $5 \mathrm{~min}$ of HUT or a stable BP without a decrease or recovery of $\geq 10 \mathrm{mmHg}$ during the initial 5 min of HUT, followed by a recovery of $\geq 15 \mathrm{mmHg}$ between 5 and $10 \mathrm{~min}$ of HUT), and transient orthostatic hypotension ( $\mathrm{tOH}$, defined as a recovery of $\geq 10 \mathrm{mmHg}$ in BP to a level that no longer satisfies the definition of $\mathrm{OH}-$ i.e., within $20 \mathrm{mmHg}$ of baselinewithin 5 min of tilting).

Among the 336 patients analyzed, $\mathrm{sOH}(43 \%)$ was the most commonly seen pattern, followed by pOH (25.2\%), tOH (17.9\%), and OHpr (13.9\%). Most of the patients in all categories had neurogenic $\mathrm{OH}$, defined as $\mathrm{OH}$ with a compensatory increase in HR of $<15$ beats per minute. Patients with pOH had both the largest decrease in systolic BP during HUT and the greatest likelihood of developing symptoms that resulted in termination of the tilt table test. Additionally, patients with sOH had lower heart rate variability during deep breathing and a smaller Valsalva ratio than patients with $\mathrm{pOH}$, suggestive of more generalized/severe cardiovascular autonomic failure. The authors postulate that the $\mathrm{SOH}$ pattern may be associated with an increased risk of cardiovascular morbidity and mortality.

Thirty-two percent of patients had sOH patterns during the first 5 min but their BP changed significantly over the subsequent 5 min. Those of us who perform HUT in our practice should note this, as it may be useful to extend tilt beyond $5 \mathrm{~min}$ rather than ending the study early, even if patients exhibit clear $\mathrm{OH}$ within the first 3 min of HUT. The limitations of this report include its retrospective nature. As a result, comorbidities such as cardiac disease were not controlled for, and this may have influenced the results. The authors used manual $\mathrm{BP}$ measurements and not beat-to-beat analysis, so transient $\mathrm{OH}$ could not be captured. Nonetheless, these differences in $\mathrm{OH}$ patterns might reflect different underlying pathological mechanisms, and this is a topic that deserves future longitudinal studies.

\section{Syncope and presyncope: diagnosis or misdiagnosis?}

Syncope can be caused by numerous conditions, which may be benign (e.g., vasovagal syncope) or deadly (e.g., autonomic failure in multiple system atrophy). Often, when patients go to the emergency room after a syncopal episode, the emergency physician is faced with the task of distinguishing these multiple entities with few historical clues or objective findings to go by. After acute, life-threatening causes have been excluded (e.g., pulmonary embolism, myocardial infarction), many patients are discharged from the emergency department without a clear diagnosis. Orthostatic $\mathrm{BP}$ is typically measured with an arm cuff, which may fail to capture a rapid reduction in $\mathrm{BP}$ (as occurs in initial $\mathrm{OH}$ or in vasovagal syncope). In the April issue of the Journal of Emergency Medicine, van Wijnen et al. [6] utilized beat-to-beat plethysmography BP readings to determine the frequencies of different orthostatic BP recovery patterns in patients presenting to the emergency room with syncope or presyncope, and hypothesized that not all BP pathology can be captured with the intermittent BP measurements obtained using the oscillometric BP arm cuff that is the current standard of care.

The authors recruited all consecutive adult patients with syncope or presyncope older than 18 years who visited the emergency room. Patients were excluded if they were not able to stand for $5 \mathrm{~min}$ or if they were hemodynamically unstable. Syncope was defined as a transient loss of consciousness due to transient global cerebral hypoperfusion, characterized by a rapid onset, a short duration, and spontaneous complete recovery. Presyncope was defined as the feeling of almost losing consciousness with similar prodromal symptoms to syncope. BP was measured continuously during $5 \mathrm{~min}$ of supine rest and continuously during $5 \mathrm{~min}$ of active standing. Patients were also asked if they experienced any symptoms of orthostatic intolerance during standing. These BP measurements were not available to the attending 
at the time of patient discharge from the emergency room, and the discharge diagnosis and BP results were reconciled after the patient had received the presumptive diagnosis.

Of the 111 patients selected for final analysis, the median age was $63 \pm 30$ years, $51 \%$ were male, $69 \%$ presented with syncope, and $41 \%$ with presyncope. Among these 111 patients, $57 \%$ had a normal BP recovery, $7 \%$ had initial $\mathrm{OH}, 13 \%$ had delayed $\mathrm{OH}, 19 \%$ had classic $\mathrm{OH}$, and $4 \%$ had vasovagal syncope. Seven out of 45 (16\%) patients initially diagnosed with vasovagal syncope by the attending physician actually had classic $\mathrm{OH}$ based on continuous BP measurement. In patients diagnosed with $\mathrm{OH}$ by the attending physician, 11/18 (61\%) had this diagnosis confirmed by beat-to-beat BP analysis. Of those patients diagnosed with unexplained syncope on discharge, beat-to-beat $\mathrm{BP}$ analysis revealed delayed $\mathrm{OH}$ in $21 \%$, initial $\mathrm{OH}$ in $14 \%$, and classic $\mathrm{OH}$ in $6 \%$. The other patients (a noteworthy $41 \%$ ) had a normal BP response.

The limitations of this study include the relatively small number of patients and the fact that the beat-to-beat BP measurements were not correlated with arm cuff oscillometric measurements, as different physicians performed either on different occasions. Nonetheless, the results are very interesting. The discrepancy between the BP results suggests that either the cuff BP measurements were not performed properly, the emergency room doctor did not interpret the arm cuff BP data properly, or beat-to-beat BP readings are significantly more accurate. An additional question is whether having beat-to-beat BP cuffs in the emergency room is cost-effective. An accurate classification of syncope in the emergency setting is of crucial importance given that it can be quite challenging to relabel the disorder of the patient once a diagnosis has been given.

Funding None.

\section{Compliance with ethical standards}

Conflict of interest The authors declare that they have no competing interests.

\section{References}

1. Damla O, Altug C, Pinar KK, Alper K, Dilek IG, Kadriye A (2018) Heart rate variability analysis in patients with multiple sclerosis. Mult Scler Relat Disord 24:64-68

2. Adamec I, Crnošija L, Junaković A, Krbot Skorić M, Habek M (2018) Progressive multiple sclerosis patients have a higher burden of autonomic dysfunction compared to relapsing remitting phenotype. Clin Neurophysiol 129:1588-1594

3. Videira G, Castro P, Vieira B et al (2016) Autonomic dysfunction in multiple sclerosis is better detected by heart rate variability and is not correlated with central autonomic network damage. J Neurol Sci 367:133-137

4. Freeman R, Wieling W, Axelrod FB et al (2011) Consensus statement on the definition of orthostatic hypotension, neurally mediated syncope and the postural tachycardia syndrome. Clin Auton Res 21:69-72

5. Seok HY, Kim YH, Kim H, Kim B-J (2018) Patterns of orthostatic blood pressure changes in patients with orthostatic hypotension. $\mathrm{J}$ Clin Neurol 14:283

6. van Wijnen VK, Ten Hove D, Gans ROB et al (2018) Orthostatic blood pressure recovery patterns in suspected syncope in the emergency department. Emerg Med J 35:226-230 